\title{
Dynamics of Complexation of a Charged Dendrimer by Linear Polyelectrolyte: Computer Modelling
}

\author{
Sergey Lyulin, ${ }^{1 *}$ Anatolij Darinskii, ${ }^{1}$ Alexey Lyulin ${ }^{2}$ \\ 1 Institute of Macromolecular Compounds, Russian Academy of Sciences, Bolshoj \\ Prospect 31, St. Petersburg, 199004, Russia; Fax: +7 812 3286869; email: \\ serge@macro.ru \\ 2 Group Polymer Physics, Eindhoven Polymer Laboratories and Dutch Polymer \\ Institute, Technische Universiteit Eindhoven, P.O. Box 5135600 MB Eindhoven, the \\ Netherlands; Fax: +31 40 2445253; email: a.v.lyulin@tue.nl
}

(Received: 9 July, 2007; published: 5 September, 2007)

\begin{abstract}
Brownian-dynamics simulations have been performed for complexes formed by a charged dendrimer and a long oppositely charged linear polyelectrolyte when overcharging phenomenon is always observed. After complex formation the orientational mobility of the individual dendrimer bonds, the fluctuations of the dendrimer size, and the dendrimer rotational diffusion have been simulated. Corresponding relaxation times do not depend on the linear-chain length in a complex and are close to those for a single neutral dendrimer. At the same time fluctuations of the size of a complex are completely defined by the corresponding fluctuations of a linear polyelectrolyte size. Adsorbed polyelectrolyte practically does not feel the rotation of a dendrimer; simulated complexes may be considered as nuts with light core (dendrimer) and heavy shell (adsorbed linear polymer); the electrostatic contacts between dendrimer and oppositely charged linear polymer are easily broken due to the very fast dendrimer-size fluctuations.
\end{abstract}

\section{Introduction}

The unique architecture and monodispersity of dendrimers allow their principle use as molecular cages for a direct transport of different guest molecules like drugs and DNA [1-4]. Complexes of dendrimers with these compounds in water solutions at neutral or low $\mathrm{pH}$ are stabilized mainly due to the electrostatic forces. In this case host and guest molecules and macromolecules possess charges of the opposite sign. Several experimental studies $[5,6,7]$ clarify general structure of dendrimerlinear polyelectrolyte (PE) complexes. In particular, atomic-force-microscopy data [8] and titration combined with spectroscopy [9] indicate that DNA macromolecules wrap around charged dendrimer-like polymers. Recently these experimental results were confirmed by the fully-atomistic molecular-dynamics (MD) simulations of Maiti and Bagchi [10]. Nevertheless, the physical properties of charged dendrimers in complexes with oppositely charged linear PE have been poorly understood. Theoretical consideration of such systems is restricted by their extreme complexity; in this case the use of computer-simulation methods becomes inevitable. First computer simulations of complexes formed by dendrimers with charged terminal groups and oppositely charged linear chains have been performed by Welch and Muthukumar [11] by Monte Carlo (MC). In their study only equilibrium characteristics, such as radius of gyration and a radial monomer density of the complex have been studied, although some suggestions about the mechanism of the relative-to-the- 
dendrimer linear-chain motion have been made [11]. Useful information about the dynamics of complexes can be obtained via MD and Brownian dynamics (BD) simulations. Very detailed MD simulations allow calculations of relatively short-time dynamic characteristics. The coarse-grained BD simulation methods help to study the long-range complex dynamics. Such a simulation was performed in our previous study [12] for complexes formed by rather long linear PE chains and oppositely charged dendrimers. The structure and the equilibrium properties of a dendrimer and a PE in a complex have been investigated. In particular, the overcharging effect was established for the system: the total number of the charged linear-chain monomers adsorbed onto an oppositely charged dendrimer exceeds the number that is necessary for dendrimer neutralization. In the present paper we focus mainly on dynamical properties of the components of a complex formed by a dendrimer and a rather long polymer chain, i.e. when the overcharging phenomenon is always observed. For both dendrimer and linear PE the toy freely-joint models have been used, which ignore the chemical details of the structure and the difference in the stiffness of the components. However, the goal of our study is to shed light on the fundamental aspects of the segmental mobility influenced by the complexation. Further detalization of the models will be, of course, necessary to distinguish between such a generic behaviour and chemistry-specific details.

Experimentally the information about the segmental relaxational properties which is the subject of the manuscript, can be obtained by using labeling of the dendrimer groups or PE monomers. The most applicable methods in this respect are neutron scattering as well as luminescence, NMR, and dielectric relaxation.

The paper is organized as follows. First, the model and the simulation algorithm are reported. Then different time-scale relaxational processes in a charged dendrimer after complexation with a PE chain are discussed and compared with those for a single dendrimer. Fluctuations of the dendrimer size, the rotational mobility of a dendrimer in a complex, and orientational mobility of the individual bonds have been reported. Dynamics of the chain-size fluctuations is discussed as well as the motion of the linear PE relative to the dendrimer. Finally, some conclusions are drawn.

\section{Model of a Complex}

As in the previous studies [12-16] we consider a bead-rod freely-joint model of a dendrimer and an oppositely charged linear PE chain, Fig. 1. The dendrimer is represented as a system of beads connected by rigid rods of length $l$ meaning that the spacer length between branching points in a dendrimer is equal to one chain bond. Dendrimers with a three-functional core and three-functional groups are studied. The $g=0$ dendrimer consists of 4 beads including the core. The total number $N$ of beads in a generation- $g$ dendrimer is calculated as

$$
N=3\left(2^{g+1}-1\right)+1
$$

In the present simulations we consider the case where all $N_{T}$ terminal beads of a dendrimer are charged with the same charge $+e$. Such a situation is realized, for example, for PAMAM and other dendrimers in water solutions at neutral $\mathrm{pH}[10,17$, 18]. The linear-polyelectrolyte chain is represented as a system of $N_{c h}$ beads, possessing the opposite charge $-e$, and connected by rigid bonds of the same length l. For both dendrimer and linear PE no valence- and torsional-angle interactions are taken into account. 


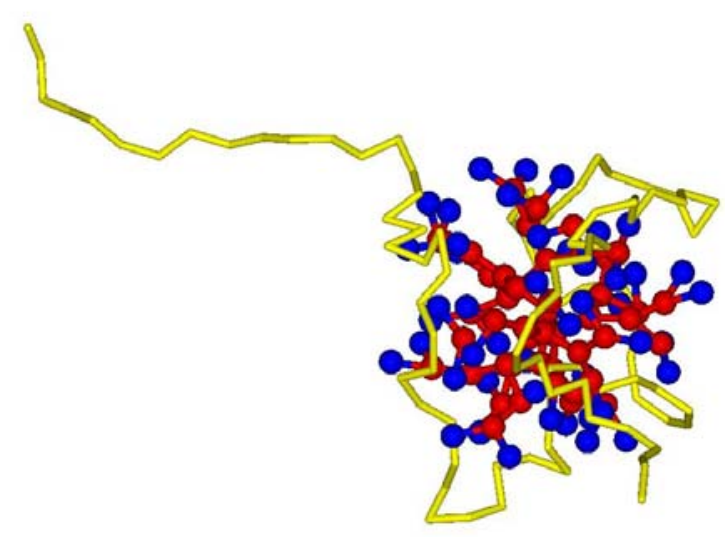

Fig. 1. Freely-joint bead-rod model employed in this study. As an example typical snapshot for a $g=4$ positively-charged dendrimer and a $N_{c h}=90$ negatively-charged linear PE chain is shown. Charged beads of the dendrimer are colored by blue.

All the non-bonded beads in a dendrimer - PE complex interact via the modified Lennard-Jones potential in which the attractive term is omitted

$\widetilde{U}_{L J}\left(r_{i j}\right)=4 \varepsilon_{L J}\left[\left(\frac{\sigma}{r_{i j}}\right)^{12}-\left(\frac{\sigma}{r_{\text {cut }}}\right)^{12}\right], \quad r<r_{\text {cut }}$

$\tilde{U}_{L J}\left(r_{i j}\right)=0, \quad r \geq r_{\text {cut }}$

here $r_{i j}$ is the distance between $i$-th and $j$-th beads, $\varepsilon_{L J}$ and $\sigma$ are the characteristic energy and length parameters, and $r_{c u t}$ is the cut-off distance, $r_{c u t}=2.5 \sigma=2 l$. This potential corresponds to the case of the athermal solvent. The values $\sigma=0.8 \mathrm{l}$ and $\varepsilon_{L J}=0.3 k_{B} T$ have been taken from our previous studies [12-14], where $k_{B}$ is the Boltzmann constant.

The $j$-th charged bead interacts with all other charged beads via the Debye-Hückel potential (electrostatic screened Coulomb potential)

$\frac{U_{j}^{C}}{k_{B} T}=\lambda_{B} \sum_{i} \frac{\exp \left(-k r_{i j}\right)}{r_{i j}}$

where $r_{i j}$ is the distance between the charged beads $i$ and $j, \lambda_{B}$ is the Bjerrum length describing the strength of the Coulomb interactions in a medium with dielectric constant $\widetilde{\varepsilon}$

$\lambda_{B}=\frac{e^{2}}{4 \pi \tilde{\varepsilon} k_{B} T}$

The value of $\lambda_{B}$ in water at room temperature is $7.14 \AA$ and is close to the segment length for a flexible polymer. Therefore, we put $\lambda_{B}=l$ without much practical loss of generality. The Debye screening constant in eq 3 is defined as

$k^{2}=4 \pi \lambda_{B} \sum_{i} z_{i}^{2} c_{i}$ 
where $c_{i}$ is the concentration of $i$-th ion and $z_{i}$ is its valence. The main effect of the electrostatic interactions should be expected when the Debye length exceeds the dendrimer size. In the present study the sufficiently large value of $r_{D}=k^{-1}=8.96 \mathrm{l}$ has been chosen (which corresponds to $2.2 \mathrm{mM}$ aqueous salt concentration at $25^{\circ} \mathrm{C}$ ), as was used by the authors previously for simulations of single charged dendrimers [1314]. For the complexes where the dendrimer charge is compensated by the charge of the linear chain the counterions condensation should be small and is not taken into account in the present study.

All simulations have been carried out for the complexes with $g=3\left(N=46, N_{T}=24\right)$ and $g=4\left(N=94, N_{T}=48\right)$ dendrimers. In such complexes the total linear-PE charge $N_{\text {ch }}$ is equal to or exceeds the charge of an individual dendrimer, i.e. $60 \geq N_{c h} \geq 24$ in complexes with $g=3$ dendrimers and $90 \geq N_{c h} \geq 48$ in complexes with $g=4$ dendrimers.

\section{Simulation Algorithm}

In the present study the hydrodynamically impenetrable model has been chosen for the simulated complexes, and hydrodynamic interactions $(\mathrm{HI})$ between different beads have been taken into account explicitly. All beads of a complex are characterized by the friction coefficient $\zeta$. The finite-difference numerical scheme implemented here is based on the Ermak-McCammon equation [12-16, 19].

$$
\vec{r}_{i}=\vec{r}_{i}^{0}+\frac{\Delta t}{k_{B} T} \sum_{j} D_{i j}^{0} \vec{F}_{j}^{0}+\vec{\Phi}_{i}^{0}(\Delta t)
$$

where $\vec{r}_{i}^{0}$ is the position vector of the $i$-th particle ( $i=0$ corresponds to the core) before the integration step, $T$ is the absolute temperature, $D_{i j}^{0}$ is the diffusion tensor, and $\Delta t$ is the integration step. Upper index 0 means the value of the variable before the integration step.

The solvent is considered as a structureless viscous continuum with chain-solvent collisions mimicked by a random force (white noise) $\vec{\Phi}^{0}$,

$\left\langle\vec{\Phi}^{0}\right\rangle=0$

$<\vec{\Phi}_{i}^{0}(\Delta t) \cdot \vec{\Phi}_{j}^{0}(\Delta t)>=2 \Delta t D_{i j}^{0}$

In eq 6 the complete force $\vec{F}_{j}^{0}$ acting on the $j$-th bead is the sum of the forces due to the rigid constraints and potential forces:

$$
\vec{F}_{j}^{0}=-\sum_{k=1}^{N} \mu_{k}\left(\frac{\partial v_{k}}{\partial \vec{r}_{j}}\right)_{r_{0}}-\partial \tilde{U}_{L J} / \partial \vec{r}_{j}^{0}-\partial U_{j}^{C} / \partial \vec{r}_{j}^{0}
$$

where $v_{k}=\frac{1}{2}\left(\vec{r}_{k+1}-\vec{r}_{k}\right)^{2}-l^{2}=0$ is the equation for the $k$-th rigid constraint, $\mu_{k}$ is the corresponding Lagrange multiplier, and $\vec{r}_{j}^{0}$ is the position vector for the $j$-th bead before a time step $\Delta t$. 
$\mathrm{HI}$ in the system are taken into account with the help of the Rotne-Prager-Yamakawa tensor $[14-16,20]$. The diagonal elements of the diffusion tensor $D_{i i}$ are defined as

$D_{i i}^{(\alpha \beta) 0}=\left(k_{B} T / \zeta\right) \delta_{\alpha \beta}$

where $\alpha, \beta$ are Cartesian components, $\delta_{\alpha \beta}$ is the Kronecker symbol. Non-diagonal elements of the diffusion tensor are represented as

$D_{i j}^{\alpha \beta}=h(\pi / 3)^{1 / 2}\left(3 k_{B} T / 4 \zeta\right)\left(l / R_{i j}\right)\left[\left(\delta_{\alpha \beta}+\frac{R_{i j}^{a} R_{i j}^{\beta}}{R_{i j}^{2}}\right)+\frac{2 a^{2}}{3 R_{i j}^{2}}\left(\delta_{\alpha \beta}-\frac{3 R_{i j}^{a} R_{i j}^{\beta}}{R_{i j}}\right)\right]$

for $R_{i j}=\left|\vec{R}_{i j}\right|=\left|\vec{r}_{i}-\vec{r}_{j}\right| \geq 2 a$

$D_{i j}^{\alpha \beta}=\left(k_{b} T / \zeta\right)\left[\left(1-\frac{9 R_{i j}}{32 a}\right) \delta_{\alpha \beta}+\left(\frac{3}{32 a}\right) \frac{R_{i j}^{a} R_{i j}^{\beta}}{R_{i j}}\right]$ for $R_{i j}<2 a$

where $R_{i j}$ is the distance between $i$-th and $j$-th beads with the hydrodynamic radius $a$. The strength of the $\mathrm{HI}$ is defined by the parameter $h=(3 / \pi)^{1 / 2} \zeta /\left(6 \pi \eta_{s} l\right)$, where $\eta_{s}$ is the solvent viscosity. As previously, the value of parameter $a$ was chosen as $a=0.257 l[14,15]$.

In the present study we use dimensionless quantities where length $l$, energy $k_{B} T$, time $\zeta 1^{2} / k_{B} T$, and translational friction of a single bead $\zeta=6 \pi \eta_{s} a$ are set to unity. The dimensionless integration step is equal to $\Delta t=10^{-4}$. This value of $\Delta t$ was chosen in order to have the maximum displacement of a bead less than $10 \%$ of the bond length. The SHAKE algorithm [21] with relative tolerance of $2 \times 10^{-6}$ is used to maintain a fixed bond length.

The initial configuration of a dendrimer is built using a procedure proposed by Murat and Grest [22]. The adding of the linear chain has been described in our previous publications [12]. The initial configuration of each complex is equilibrated for seven runs of $2 \times 10^{6}$ steps each, then seven production runs of $2 \times 10^{6}$ time steps are performed. During each run the instantaneous values of the squared radius of gyration $R_{g}^{2}$ are calculated for the whole complex, an individual linear PE in a complex, and an individual dendrimer in a complex. Observed fluctuations of the gyration radius around its average value indicated well-equilibrated states for all studied samples.

\section{Results and discussion}

\section{Relaxation Processes of a Dendrimer and a Linear PE in a Complex}

The mean-square radius of gyration $\left\langle R_{g}^{2}\right\rangle$ is used as a measure for both a dendrimer and a linear-PE size in the simulated complex, and for a size of the complex as a whole $[12,13]$. We start below with the discussion of fluctuations of the dendrimer size in a complex. These fluctuations can be characterized by the following autocorrelation function $(\mathrm{ACF}) C_{R_{g}^{2}}(t)$ of the corresponding gyration radius 
$C_{R_{g}^{2}}(t)=\frac{\left\langle R_{g}^{2}(0) R_{g}^{2}(t)\right\rangle-\left\langle R_{g}^{2}\right\rangle^{2}}{\left\langle R_{g}^{4}\right\rangle-\left\langle R_{g}^{2}\right\rangle^{2}}$

For an individual dendrimer in a complex the relaxation of the ACF $C_{R_{g}^{2}}(t)$ occurs due to the internal motions of dendritic monomers only; the global rotation of a dendrimer as a whole does not contribute to this relaxation. Time dependences of the corresponding ACFs are shown in Fig. 2. As for a single neutral dendrimer [14] the time dependence of $\log C_{R_{g}^{2}}(t)$ for charged dendrimers in complexes with different linear PEs is close to linear, i.e. these ACFs decay exponentially. The time $\tau_{R_{g}^{2}}$ at which each ACF decays in e times has been taken as a measure for the rate of the corresponding relaxation.

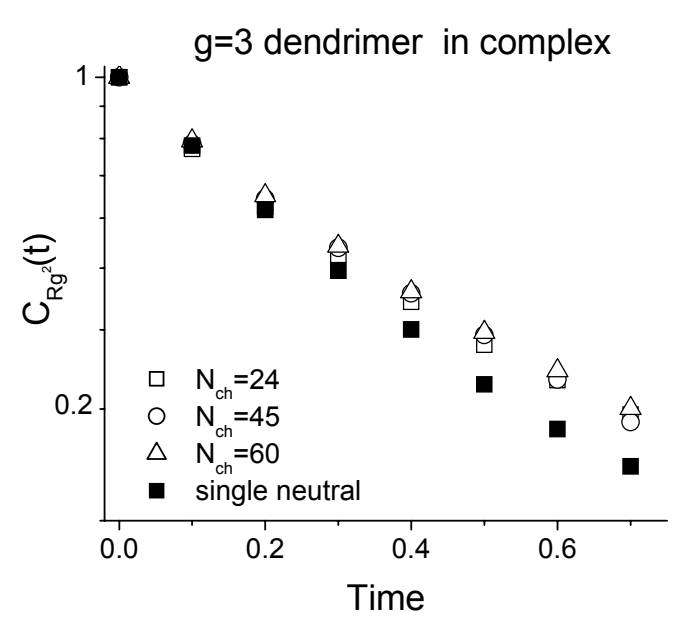

(a)

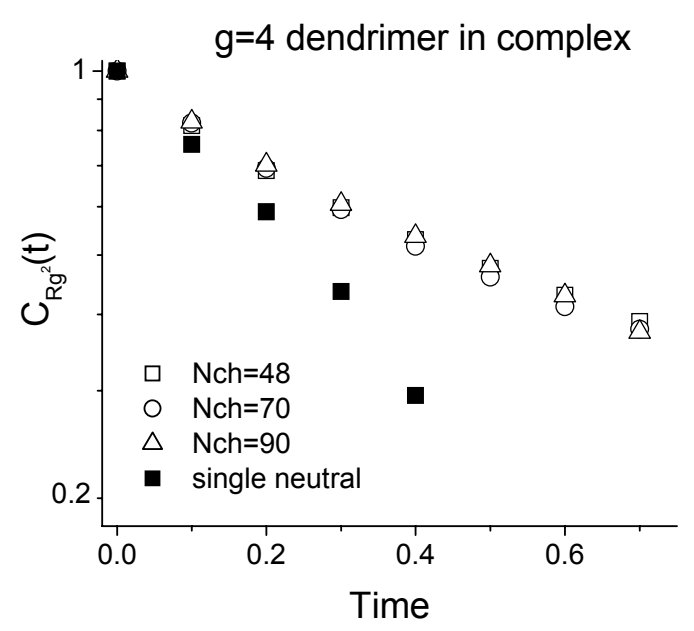

(b)

Fig. 2. Time dependence of the autocorrelation functions for the gyration radius of a) $g=3$ and b) $g=4$ dendrimer in complexes with different linear PEs.

The chain-length dependence of $\tau_{R_{g}^{2}}$ for different complexes is shown in Fig. 3a together with the corresponding times for a linear chain in a complex and for a complex as a whole. Our previous simulations of both neutral and charged single dendrimers have shown [14] that the value of the corresponding relaxation time $\tau_{R_{g}^{2}}$ is insensible to the dendrimer size. To explain this effect the dendrimer was represented as a viscoelastic spherical body [14]. In such a model only elastic coefficient and monomer friction influence the relaxation time $\tau_{R_{g}^{2}}$. These two parameters have similar dendrimer-size dependence, therefore the increase of the dendrimer size (i.e. number of generations) does not change the relaxation time $\tau_{R_{g}^{2}}$. Calculated values of $\tau_{R_{g}^{2}}$ in the present study for dendrimers in complexes with different linear chains are also very close to each other, because the change of the chain length does not lead to the change of the dendrimer elastic and friction coefficients. 


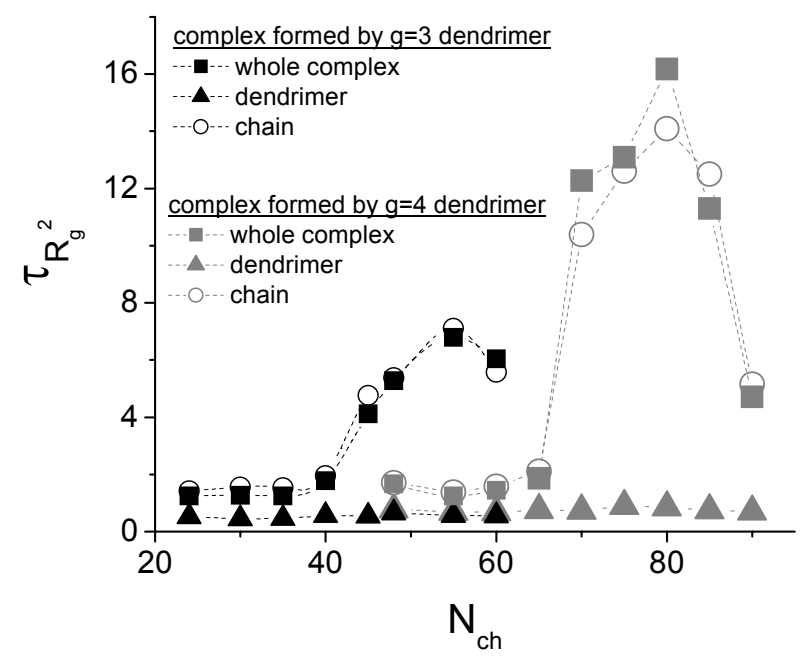

(a)

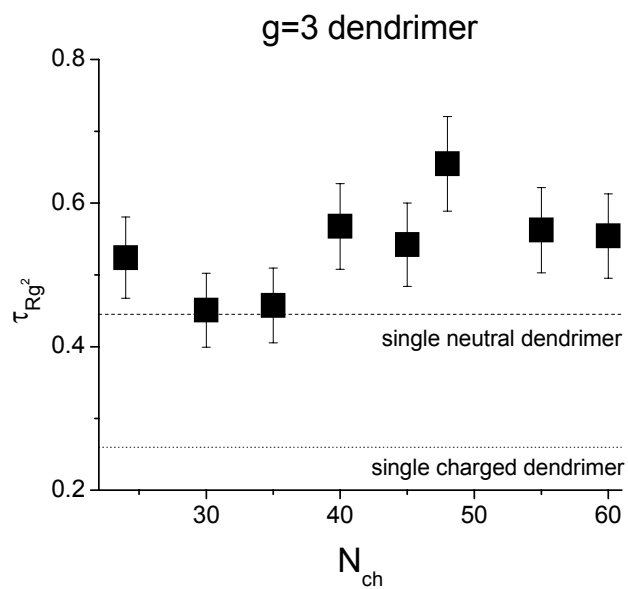

(b)

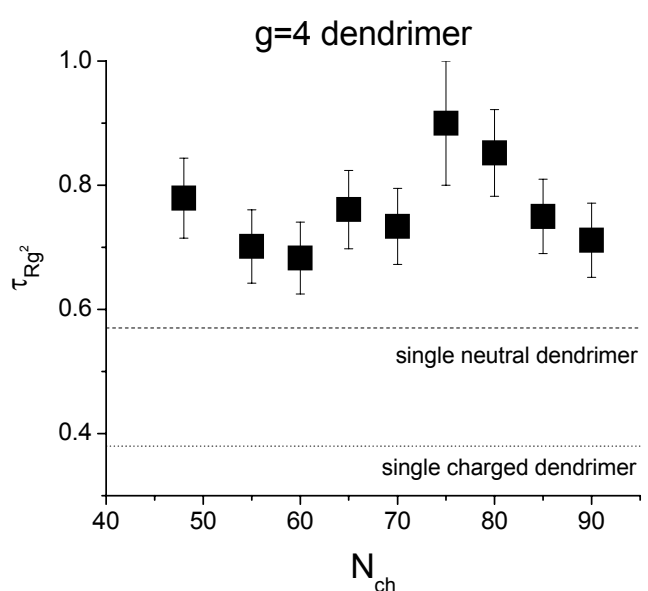

(c)

Fig. 3. a) Dependence of the relaxation times $\tau_{R_{g}^{2}}$ of the gyration radius for a complex as a whole, a linear chain and a dendrimer in a complex. Dashed lines are drawn as guides to the eye. Relaxation times $\tau_{R_{g}^{2}}$ for b) $g=3$ and c) $g=4$ dendrimers in complexes are shown in comparison with the corresponding values for a single neutral and a single charged dendrimer.

We have also shown previously [12] that the internal structure of the charged dendrimer in a complex is similar to that for a single neutral dendrimer. This observation explains the constant elasticity of the dendrimer in complexes with linear PEs of different size. Fluctuations of a gyration radius of a charged dendrimer in a complex do not depend on the PE length, and are very close to the values of the corresponding fluctuations for a single neutral dendrimer [13]. It means that the charged dendrimer in a complex is not sensitive to the length of the adsorbed PE, and the "weighing" of the dendrimer by the PE does not occur. However, for all simulated complexes the relaxation of a dendrimer size is slower than that for a corresponding single charged or single neutral dendrimer, [14], Fig. 3b), 3c). 
As was shown by us earlier [12] the significant changes of the structural properties of the complexes upon increase of the PE length occur due to the structural changes of the added polyelectrolyte. How are the dynamic properties influenced by the complexation? First, the relative fluctuations $\Delta$ of the chain radius of gyration $\left\langle R_{g}^{2}\right\rangle$ have been calculated, Fig. 4:

$\Delta\left\langle R_{g}^{2}\right\rangle_{\text {chain }}=\frac{\left\langle R_{g}^{4}\right\rangle_{\text {chain }}-\left\langle R_{g}^{2}\right\rangle_{\text {chain }}^{2}}{\left\langle R_{g}^{2}\right\rangle_{\text {chain }}^{2}}$

At small PE length $N_{c h} \sim N_{n}$, which is necessary for the dendrimer electro neutralization, the value of $\Delta\left\langle R_{g}^{2}\right\rangle_{\text {chain }}$ is rather small $(\sim 0.005)$ and is equal to that for a single dendrimer [13]. At $N_{c h}>N_{n}$ the fluctuations increase significantly and have non-monotonic behavior. The maximum of these fluctuations as a function of $N_{c h}-N_{n}$ is observed at $N_{c h}-N_{n} \sim 30$ for all complexes. It corresponds to the chain length $N_{c h} \sim 55$ for the complexes formed by $g=3$ dendrimer and $N_{c h} \sim 80$ for the complexes formed by $g=4$ dendrimer, i.e. exactly where the overcharging of the dendrimer by oppositely charged PE is at its maximum [12]. At higher values of $N_{c h}$ the overcharging (and fluctuations $\Delta\left\langle R_{g}^{2}\right\rangle$ ) decreases and the conformation of the PE with rather long tail becomes preferable energetically. For a complex formed by the impenetrable charged sphere and an oppositely-charged linear PE both theory and computer simulation consider the tail appearance as the first order phase transition $[23,24]$. It is well known [25] that such a transition is accompanied by the increase of fluctuations and slowing down of relaxation times. As was shown by us earlier [12] the corresponding transition in the complexes formed by the charged dendrimer is not that sharp as for a hard sphere. However, the characteristic features of the phase transition remain valid [12].

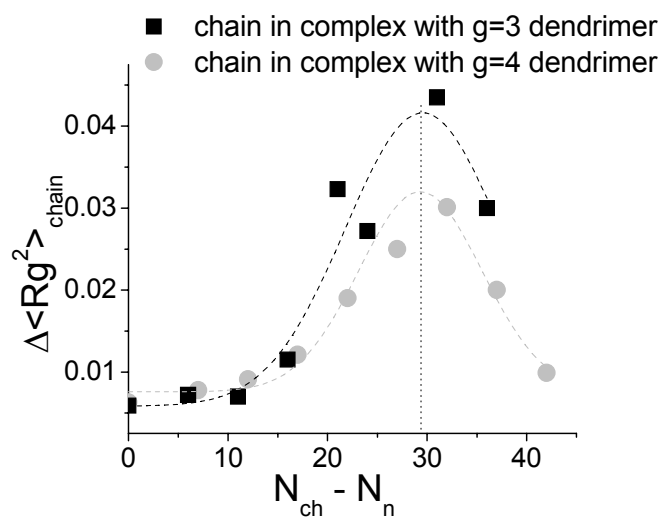

Fig. 4. Chain-length dependence of the relative fluctuations of the gyration radius $R_{g}^{2}$ for the linear PE in complexes with $g=3$ and $g=4$ dendrimers. Dashed lines are drawn as guides to the eye. Vertical dash line shows the position of the maximum.

As for an individual dendrimer in a complex, the ACFs for the PE radius of gyration also decay exponentially. Corresponding relaxation times $\tau_{R_{g}^{2}}$ are much larger (Fig. 3) as compared to those for an individual dendrimer in a complex, and have non- 
monotonic behaviour similar to the behavior of the relative fluctuations of the chain radius of gyration.

\section{Fluctuations of a Complex Size}

We also calculated relaxation times $\tau_{R_{g}^{2}}$ for a whole complex. The dependence of $\tau_{R_{g}^{2}}\left(N_{c h}\right)$ for a complex as a whole is very similar to the corresponding dependence for a linear PE in a complex, Fig. 3. It means that the dynamics of the fluctuations of the complex' size is determined mainly by the fluctuations of the PE size. Corresponding relaxation times for a dendrimer are much smaller, and do not contribute to the complex-size relaxation.

\section{Rotation of a Dendrimer as a whole}

Rotation of a dendrimer as a whole in a complex is characterized by the following autocorrelation function

$$
C_{r o t}(t)=\frac{1}{N_{T}} \sum_{i=1}^{N_{T}}\left\langle\vec{e}_{g}^{i}(0) \vec{e}_{g}^{i}(t)\right\rangle
$$

for the unit vector $\vec{e}_{g}^{i}=\frac{\vec{Q}_{g}^{i}}{\left|\vec{Q}_{g}^{i}\right|}$ directed along the position vector $\vec{Q}_{g}^{i}$ for the $i$-th dendrimer terminal monomer with respect to the core,

$\vec{Q}_{g}^{i}=\vec{r}_{i}-\vec{r}_{0}$

where $\vec{r}_{i}$ represents the position of $i$-th terminal monomer and $\vec{r}_{0}$ corresponds to the core. $C_{r o t}(t)$ decays exponentially and can be characterized by the single e-time decay relaxation time $\tau_{\text {rot }}$. The values of $\tau_{\text {rot }}$ are slightly larger than the corresponding values for a single neutral dendrimer.

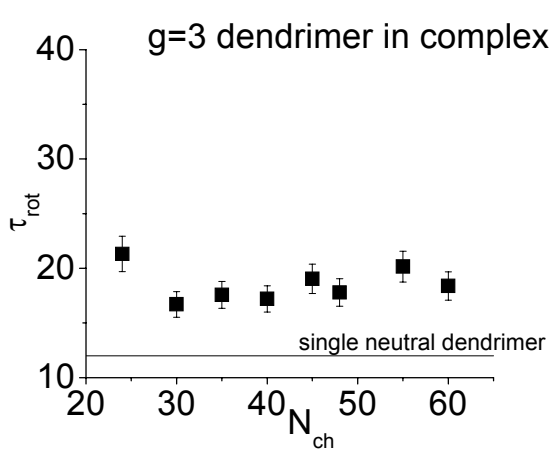

(a)

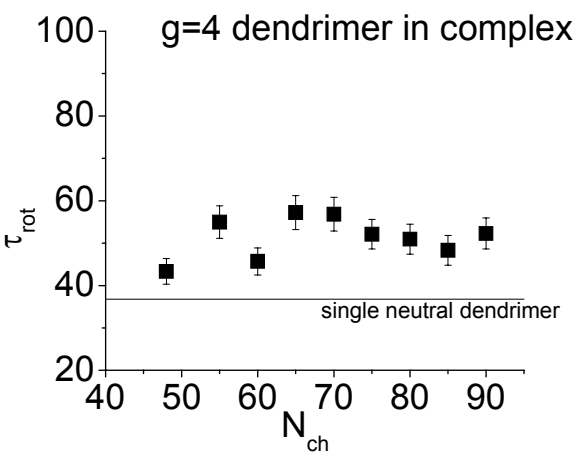

(b)

Fig. 5. PE-length dependence of the relaxation times $\tau_{\text {rot }}$ characterizing the rotation of (a) $g=3$ dendrimer and (b) $g=4$ as a whole in a complex.

For a solid spherical body in a viscous medium $\tau_{\text {rot }} \sim R^{3}$, where $R$ is the radius of a solid sphere. For a non-draining model of a single neutral dendrimer the same result 
has been obtained [14], where $R=\sqrt{\frac{5}{3} R_{g}^{2}}$. Thus, the very weak dependence of the time $\tau_{\text {rot }}$ on the linear-PE length may be due to the fact that the size of a dendrimer in complexes with different linear chains remains almost constant [12].

\section{Local Orientational Mobility}

To study the local orientational mobility of individual monomers in a dendrimer we calculated the orientational autocorrelation functions $P_{1}(t)$ for dendrimer bonds

$P_{1}(t)=\left\langle\vec{b}_{i}(0) \cdot \vec{b}_{i}(t)\right\rangle$

where $\vec{b}_{i}(t)$ is the unit vector directed along the $i$-th bond. $P_{1}(t)$ for dendrimer bonds belonging to different generations (averaged $P_{1}$ over all beads belonging to the corresponding generation) are shown in Fig. 6a for the complex formed by $g=3$ dendrimer and $N_{c h}=24$ chain. The similar picture is observed for all simulated complexes. The mobility decreases for bonds which are closer to the core. The terminal bonds are the most mobile, as also established earlier for both neutral and charged single dendrimers [14]. The orientational relaxation of dendrimer bonds is strongly influenced by the rotation of a dendrimer as a whole. The contribution of the overall dendrimer rotation increases for bonds which are closer to the core. The mobility of bonds attached to the core is the most restricted, the orientational relaxation of these bonds occurs mainly through the rotation of a dendrimer as a whole.

Figs. $6 \mathrm{~b}$ and $6 \mathrm{c}$ show the time dependence of $P_{1}(t)$ for terminal bonds of $g=3$ and $g$ $=4$ dendrimer, correspondingly, in complexes with chains of different length.

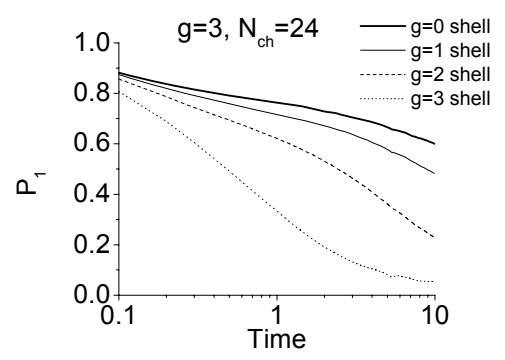

(a)

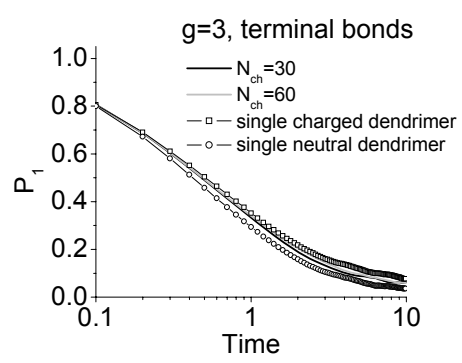

(b)

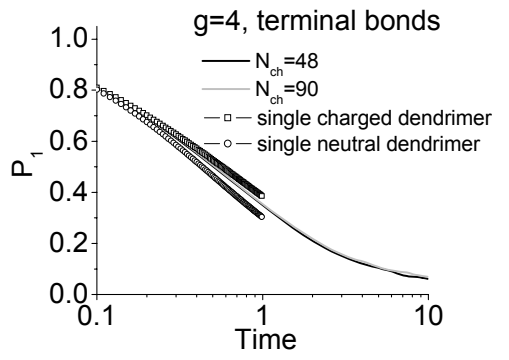

(c)

Fig. 6. a) Time dependence of $P_{1}(t)$ for the beads belonging to the different generation shells of a $g=3$ dendrimer in a complex with $N_{c h}=24$ linear chain. Time dependence of $P_{1}(t)$ for the terminal bonds for $\left.b\right) g=3$ and c) $g=4$ dendrimer.

The influence of the complex formation onto $P_{1}(t)$ relaxation of individual-dendrimer bonds occurs to be rather weak. The curves characterizing $P_{1}(t)$ relaxation of individual dendrimer bonds in complexes lie between those for a single neutral and a single charged dendrimers. The change of the linear-PE length does not influence the mobility of dendritic bonds. These results agree with the previous observations that the rotational mobility of a dendrimer as a whole is only slightly affected by the linear-polyelectrolyte adsorption. 


\section{Chain Motion Relative to the Dendrimer Rotation}

The linear-chain motion relative to the dendrimer in a complex has been studied by MC simulation of Welch and Muthukumar [11], and was based on the snapshot analysis. To characterize more quantitatively the motion of a chain relative to a dendrimer in a complex, the correlation functions $P_{1}(t)$ for unit vectors $\vec{c}_{i}$ directed from the dendrimer core to different PE monomers have been calculated,

$P_{1}^{i}(t)=\left\langle\vec{c}_{i}(0) \cdot \vec{c}_{i}(t)\right\rangle$

Fig. 7 shows the resulting ACF function $C_{\text {rel }}(t)$

$$
C_{\text {rel }}(t)=\frac{1}{N_{c h}} \sum_{i=1}^{N_{c h}} P_{1}^{i}(t)
$$

averaged over all chain monomers, together with a typical dependence $C_{\text {rot }}(t)$ (eq. 14) for a dendrimer in a complex with $N_{c h}=90$ PE.

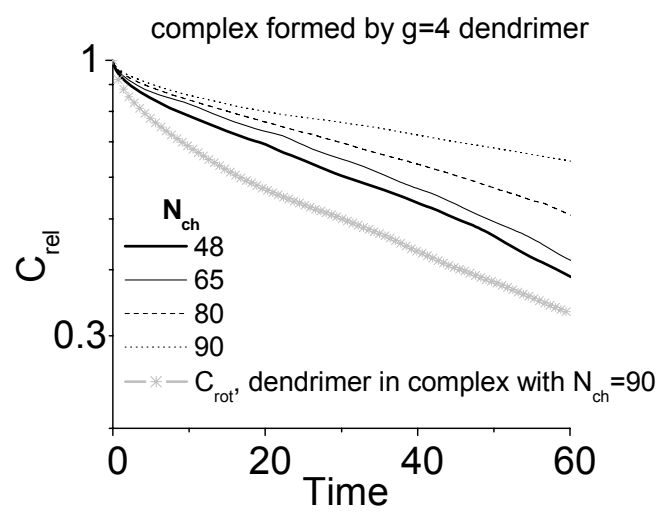

Fig. 7. Time dependence of $C_{\text {rel }}(t)$ for complexes formed by $g=4$ dendrimer.

The rotation of a dendrimer as a whole occurs faster than the rotation of PE monomers around the dendrimer core, Fig. 7. The motion of PE monomers relative to the dendrimer becomes slower when PE length increases. At the same time, as was shown earlier in the present study, the rate of the dendrimer rotation does not depend on the chain length. It means that the dendrimer rotates practically independently from the linear PE. Such a behaviour resembles the behaviour of the light kernel of a nut in a heavy shell. The weight of this "shell" increases with the PE length and the difference between rotation of the kernel and that of a shell increases. These results show that the PE chain can move rather freely on a surface of a dendrimer and its mobility is determined mainly by its own friction.

Intuitively, this result disagrees with the presence of the strong electrostatic interactions between charged dendrimer and the oppositely charged linear PE. That is why we have also estimated the average life time of a single electrostatic contact between oppositely charged beads of the linear chain and the dendrimer. PE bead is considered to be in the electrostatic contact with the charged $j$-th terminal bead of a dendrimer if the distance between their centers $r_{i j}$ satisfies the following 


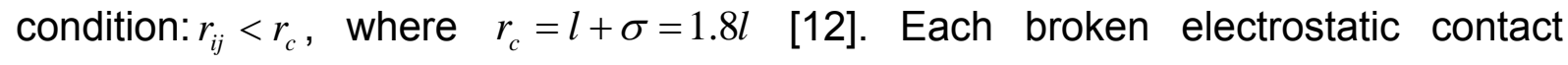
between charged PE bead and the oppositely charged dendrimer terminal bead may appear again after some time. In order to check the stability of these contacts with time the following correlation function has been calculated

$$
C_{i j}(t)=\overline{\left\langle\vec{u}_{C H}^{i} \vec{u}_{D}^{j}\right\rangle_{t}}
$$

where averaging is made over time $t, i$ and $j$ are the numbers of a PE bead and a terminal dendrimer bead, correspondingly, $\vec{u}_{C H}^{i}$ is the unit vector directed from the core of a dendrimer to the $i$-th PE bead, and $\vec{u}_{D}^{j}$ is the unit vector directed from the core of a dendrimer to the $j$-th charged dendrimer bead. This function may be considered as a "memory" function of each contact: it is close to unity at small times for beads in contact, and fluctuates around zero at large times. For the beads which are not in a contact at time $t=0$ the corresponding correlation function is always fluctuating around zero.

In Fig. 8 the time dependence of $C_{i j}^{\text {cont }}(t)$

$$
C_{i j}^{\text {cont }}(t)=\frac{1}{N_{\text {cont }}} \sum_{i, j} C_{i j}(t)
$$

is shown for the complexes formed by $g=3$ dendrimer and $N_{c h}=60$ chain, where averaging in Eq. 20 is made over all pairs formed by $i$-th and $j$-th beads which are in electrostatic contact at time $t=0, N_{\text {cont }}$ is the total number of electrostatic contacts at that time. Typical time dependence of $C_{i j}(t)$ for the pairs which are not in the electrostatic contact at time $t=0$ is also shown for all not-in-contact pairs formed by the middle chain bead (number 34 ) and charged terminal beads of a dendrimer,

$$
C_{34, j}^{\text {uncont }}(t)=\frac{1}{N_{T}-1} \sum_{j=1}^{N_{T}-1} C_{34, j}(t)
$$

$C_{34, j}^{u n c o n t}(t)$ indeed fluctuates around zero. For pairs which are in contact at time $t=0$ the corresponding function $C_{i j}^{\text {cont }}(t)$ has several relaxation regimes, see the inset to the Fig. 8, where multiple relaxation processes are clearly seen. The first process (time $t \sim 10$ ) corresponds to the time scale of the dendrimer size fluctuations. The next slower process $(t \sim 50)$ corresponds to the time scale of the rotation of the dendrimer as a whole. Thus, the break of the electrostatic contacts occurs due to the fluctuations of the dendrimer size, which is a much faster relaxation process as compared to the rotation of a dendrimer as a whole. Therefore, the rotation of a dendrimer corresponds to the times which are larger than the characteristic times necessary to break the electrostatic contacts. So, rotation of a dendrimer as a whole does not lead to the corresponding rotation of the linear PE (in such a situation the electrostatic contacts would live longer), and dendrimer rotates independently inside the "shell" formed by the linear polyelectrolyte. This result could be, of course, just a consequence of the oversimplified freely-joint model of the present study. The model which will correctly capture the friction between a PE chain and a dendrimer is a subject of our further investigation. 


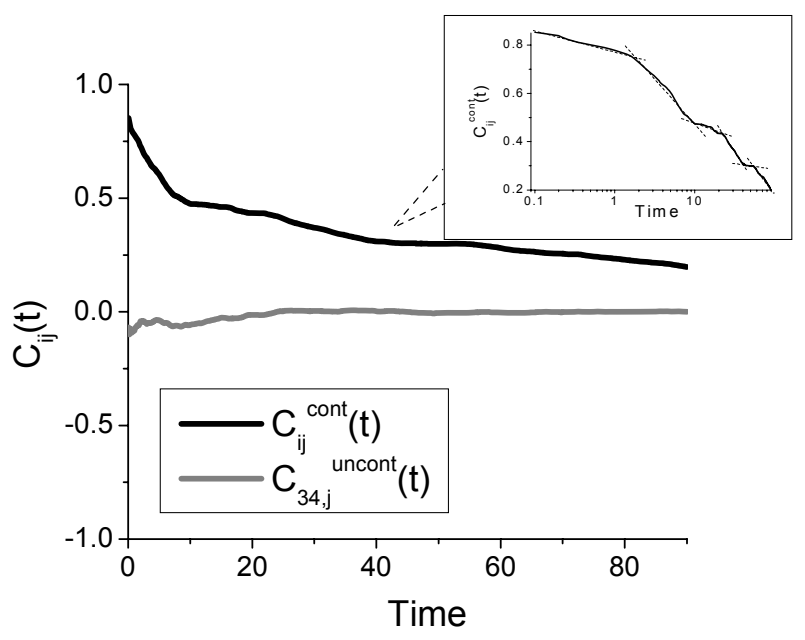

Fig. 8. Time dependence of $C_{i j}(t)$ relaxation functions. All functions correspond to the complex formed by $g=3$ dendrimer and $N_{c h}=60$ chain. Inset corresponds to the time-dependence of $C_{i j}^{\text {cont }}(t)$ in a semilog scale.

\section{Conclusions}

The dynamic properties of charged dendrimers in complexes - the dendrimer rotation as a whole, dynamics of its size fluctuations, and orientational relaxation of the individual bonds - does not change with the increase of the linear-PE length. These dependences are close to those for a single neutral dendrimer. The dynamics of the size fluctuations of the complex as a whole is defined mainly by the size fluctuations of the linear PE in a complex. The rotation of the dendrimer as a whole does not lead to the accompanying rotation of the adsorbed PE, in spite of the strong electrostatic interactions between macroion and a polyion. These interactions can be broken by the dendrimer-size fluctuations. For simulated complexes the model of a complex as a nut with light core and heavy shell may be used. It will be useful to check these conclusions further for broader range of Debye lengths and for more detailed models, i.e. taking into account the stiffness of each complex component, counterions presence and more realistic intra-molecular potentials.

\section{Acknowledgements}

This work was carried out with the financial support of RFBR grant 05-03-32450, NWO grant 047.019.001, and INTAS grant 05-109-4111 and Russian Science Support Foundation. Massive simulations have been performed using the supercomputing facilities of the Stichting Nationale Computerfaciliteiten (NCF) in Amsterdam.

\section{References}

[1] Esfand, R.; Tomalia, D.A. Drug Discovery Today. 2001, 6, 427.

[2] Patri, A.K.; Majoros, I.J.; Baker, J.R. Curr. Opin. Chem. Biol. 2002, 6, 466.

[3] Cloninger, M.J. Curr. Opin. Chem. Biol. 2002, 6, 742.

[4] Sadler, K.; Tam, J.P. J. Biotechnol. 2002, 90, 195. 
[5] Wang, Ch.; Ren, B.; Tong, Zh.; Zeng, F.; Liu, X.; Wu, Sh.; Liu, P. Eur. Polym. J. 2005, 41, 185.

[6] Miura, N.; Dubin, P.L.; Moorefield, C.N.; Newkome, G.R. Langmuir 1999, 15, 4245

[7] Kabanov, V.A.; Zezin, A.B.; Rogacheva, V.B.; Gulyaeva, Zh.G.; Zansochova, M.F.; Joosten, J.G.H.; Brackman, J. Macromolecules 1999, 32, 1904.

[8] Gössl, I.; Shu, L.; Schlüter, D.; Rabe, J. P. J. Am. Chem. Soc. 2002, 124, 6860.

[9] Kabanov, V.A.; Sergeyev, V.G.; Pyshkina, O.A.; Zinchenko, A.A.; Zezin, A.B.; Joosten, J.G.H.; Brackman, J.; Yoshikawa, K. Macromolecules 2000, 33, 9587.

[10] Maiti, P.K.; Bagchi, B. Nano Lett., 2006, 6, 2478.

[11] Welch, P.; Muthukumar, M. Macromolecules 2000, 33, 6159.

[12] Lyulin, S. V.; Darinskii, A. A.; Lyulin, A. V. Macromolecules 2005, 38, 3990.

[13] Lyulin, S. V.; Evers, L. J.; van der Schoot, P.; Darinskii, A. A.; Lyulin, A. V.; Michels, M. A. J. Macromolecules 2004, 37, 3049.

[14] Lyulin, S. V.; Darinskii, A. A.; Lyulin, A. V.; Michels, M. A. J. Macromolecules 2004, 37, 4676.

[15] Lyulin, A.V.; Davies, G.R.; Adolf, D.B. Macromolecules 2000, 33, 3294.

[16] Lyulin, A.V.; Adolf, D.B.; Davies, G.R. Macromolecules 2001, 34, 8818.

[17] a. Lee, I.; Athey, B. D.; Wetzel, A. W.; Meixner, W.; Baker, J. R. J. Macromolecules 2002, 35, 4510. b. Nisato, G.; Ivkov, R.; Amis, E. J. Macromolecules 1999, 32, 5895.

[18] van Duijvenbode, R. C.; Borkovec, M.; Koper, G. J. M. Polymer 1998, 39, 2657.

[19] Ermak, D.L.; McCammon, J.A. J. Chem. Phys. 1978, 69, 1352.

[20] Rotne, J.; Prager, S. J. Chem. Phys. 1969, 50, 4831.

[21] Ryckaert, J.-P.; Bellemans, A. Chem. Phys. Lett. 1975, 30, 123.

[22] Murat, M.; Grest, G. Macromolecules 1996, 29, 1278.

[23] Nguyen, T. T.; Shklovskii, B. I. Physica A 2001, 293, 324.

[24] Grosberg, A. Yu.; Nguyen, T. T.; Shklovskii, B. I. Reviews of Modern Rhysics 2002, 74, 329.

[25] Landau L. D., Lifshitz E.M. Statistical Physics; Pergamon: 1980. 